\title{
Chronisch-obstruktive Lungenerkrankung - Therapie
}

\author{
A. Gillissen ${ }^{1}$, W. Seeger ${ }^{2}$ \\ 1 Robert Koch-Klinik, Städtisches Klinikum »St. Georg», Leipzig \\ 2 Medizinische Klinik und Poliklinik II, Universität Gießen
}

- in die Reduktion von Risikofaktoren und

- in pharmakologische und ergänzende nicht-pharmakologische Therapiemaßnahmen. Dabei müssen klinisch die stabile und die akut exazerbierte Erkrankungsphase voneinander unterschieden werden, da sowohl die Indikation als auch die Effizienz der zur Verfügung stehenden Therapiemaßnahmen bei diesen beiden Erkrankungsformen unterschiedlich sind.

\section{Reduktion von Risikofaktoren}

Die effektivste Therapie der COPD besteht in der Reduktion von Risikofaktoren, insbesondere der Zigarettenrauchexposition. Dazu gibt es entsprechend internationalen Therapieempfehlungen mehrere Möglichkeiten, beginnend mit wiederholten Ermahnungen über Kurse und Seminare, Gesprächstherapien und auch die Pharmakotherapie (3).

\section{Pharmakotherapie der stabilen COPD}

Die Pharmakotherapie wird entsprechend des zunehmenden Schweregrades der Erkrankung intensiviert. Bisher konnte noch nicht nachgewiesen werden, dass ein medikamentöser Ansatz die langjährige Verschlechterung der Lungenfunktion bei COPDPatienten signifikant reduzieren kann, weswegen die Pharmakotherapie primär zur Reduktion der Symptome, der typischen COPD-Komplikationen und der Reduktion der Exazerbationshäufigkeit begründet ist. Die COPD-Therapie fußt im Wesentlichen auf dem Einsatz von bronchodilatativ wirkenden Medikamenten, die entweder als Bedarfsmedikation, oder als Dauermedikation verabreicht werden. Zu diesen zählen kurz- und langwirksame $\beta_{2}$-Agonisten, Anticholinergika und Methylxanthine. Risikopatienten (klinische Zeichen einer chronischen Bronchitis, bestehende Risikofaktoren, z.B. Raucher) ohne nachgewiesene Atemwegsobstruktion bedürfen keiner Pharmakotherapie. Die Vermeidung von Risikofaktoren ist allerdings angezeigt.

\section{Stufentherapieplan}

- Patienten mit einer milden Erkrankungsform (Stufe I, FEV $\geq 80 \%_{\text {Soll }}, \mathrm{FEV}_{1} / \mathrm{FVC}<70 \%$, mit und ohne Symptome) werden mit inhalativen kurzwirksamen Bronchodilatatoren bei Bedarf therapiert.

- In der moderat erkrankten COPD-Gruppe (Stufe II, FEV $<80 \%$ soll bis $\geq 30 \%, \mathrm{FEV}_{1} / \mathrm{VC}<70 \%$ mit und ohne Symptome) erfolgt eine intensivierte Therapie mit einem oder mehreren Bronchodilatatoren (kurz-/langwirksamen $\beta_{2}$-Agonisten, Anticholinergika, Theophyllin). Ergänzend sind rehabilitative Maßnahmen in den Stufen II und III indiziert (20). Inhalative Gluko-

Dtsch Med Wochenschr 2002; 127: 510-513

(c) Georg Thieme Verlag Stuttgart · New York kortikosteroide können in den Stufen II und III eingesetzt werden, sofern eine Verbesserung der klinischen Symptomatik, eine Verbesserung der Lungenfunktionsprüfung, der Lebensqualität oder der physischen Leistungsfähigkeit individuell z. B. im $1 /{ }_{4}$-Jahresabstand nachweisbar ist $(7,13)$. Inhalativ applizierte Steroide haben auch bei langjährigem Einsatz einen allenfalls geringen Effekt auf die Vermeidung bzw. Reduktion der bei dieser Erkrankung typischerweise beschleunigten Lungenfunktionsverschlechterung $(8,12,15,19,21,29,31)$. Die in vielen Therapieempfehlungen vorgeschlagene orale Kortikosteroidtherapie zur Identifikation von Steroid-Respondern wurde in den GOLD-Empfehlungen verlassen, da das Ergebnis dieses Steroid-Stoß-Tests keinen prädiktiven Wert für die Erkennung eines Steroid-Responders/Non-Responders hat. Der Stellenwert der Methylxantine wird niedrig eingeordnet (20). Eine systemische Langzeit-Steroid-Therapie wird nicht empfohlen (4).

- In der Gruppe der schwer erkrankten COPD-Patienten (Stufe III, $\mathrm{FEV}_{1}<30 \%$ des Sollwertes, mit/ohne respiratorische Insuffizienz, mit/ohne Cor pulmonale) wird die Pharmakotherapie der Stufe II durch entsprechend symptomatische Therapiemaßnahmen (z.B. Sauerstofflangzeittherapie, Behandlung des Cor pulmonale) ergänzt (Abb.1).

kurzgefasst: Die Meidung von Risikofaktoren steht an erster Stelle in der COPD-Therapie. In der Pharmakotherapie kommen in Abhängigkeit von der Erkrankungsschwere bronchodilatativ wirkende Substanzen und nur in Fällen nachgewiesener Effektivität auch inhalative Steroide in der stabilen Erkrankungsform zum Einsatz.

\section{Flankierende Maßnahmen}

Die pharmako-therapeutischen Maßnahmen werden durch ergänzende Therapien flankiert. Eine Influenza-Impfung kann die Häufigkeit schwerer Erkrankungs-/Exazerbationsformen und die Mortalität um ca. $50 \%$ senken und ist daher in allen Stufen empfohlen. Zudem wird bei Patienten ab einem Alter von 60 Jahren und einer fortgeschrittenen COPDErkrankungsform eine Pneumokokken-Impfung angeraten. Die Alpha-1-Antitrypsin-Therapie ist nur bei Patienten mit nachgewiesenem Alpha-1-Antitrypsin-Mangel indiziert. Die derzeitige Datenlage lässt keine Empfehlung zur Langzeittherapie mit Mukolytika und/oder Sekretolytika zu. Ein klinischer Effekt bei einer kurzfristigen Anwendung, z.B. bei einem bronchialen Infekt, wird diskutiert. Antitussiva, Vasodilatatoren und zentrale Atemstimulantien sind entweder kontraindiziert oder werden nicht empfohlen. Aufgrund der unsicheren Datenlage wird in keiner COPD-Therapieempfehlung der Einsatz von Antioxidantien und Immunregulatoren gut geheißen. Morphine, z.B. im Rahmen einer Exazerbation, können individuell eingesetzt werden, erfordern aber ein enges Monitoring. Nedocromil, Leukotrien-Rezeptorantagonisten oder alternative Heilmethoden (Akupunktur, Homöo- 
pathie) haben keinen signifikanten klinischen Effekt bei der COPD. Im Gegensatz dazu sind jedoch empfohlen: rehabilitative Maßnahmen in fachpneumologischen Einrichtungen, eine Sauerstofflangzeittherapie (Gabe von > 16 Std./Tag) bei Patienten in der respiratorischen Insuffizienz $\left(\mathrm{p}_{2} \leq 7,3 \mathrm{kPa} /\right.$ $55 \mathrm{mmHg})(1,5,20,32)$.

\section{Operative Verfahren}

Das Prinzip der Volumen-reduzierenden Operation besteht in der Resektion emphysematösem Lungengewebes, so dass die durch das Emphysem initial bedingte Kompression gesunden Lungengewebes reduziert und dadurch die Ventilation wieder ökonomisiert wird. Dieser Eingriff ist nur bei einem kleinen Prozentsatz der COPD-Patienten geeignet. Um den Therapieerfolg zu optimieren und Komplikationen zu reduzieren, muss die Patientenauswahl streng nach folgenden Kriterien erfolgen:

- ausreichender Karnofsky-Index,

- Fehlen gravierender Begleiterkrankungen,

- Vorhandensein bestimmter lungenfunktioneller "Grenzwerte«: $\mathrm{FEV}_{1} 20-35 \%_{\text {soll }}$, RV (Residualvolumen) $>200 \%_{\text {soll }}$, TLC $($ Totalkapazität $)>130 \%_{\text {soll }}(17,23,26)$.

Bei Patienten mit $\mathrm{FEV}_{1}<20 \%_{\text {soll }}$, homogenem Verteilungsmuster des Lungenemphysems sowie einem CO-Transferfaktor < $20 \%_{\text {soll }}$ ist dieser Eingriff nach den erst kürzlich publizierten Ergebnissen der NETT-Studie als kontraindiziert zu betrachten (18). Weitere Ausschlusskriterien sind eine Hyperkapnie $\left(\mathrm{pCO}_{2}\right.$ $>55 \mathrm{mmHg}$ ) sowie eine pulmonale Hypertonie mit einem mittleren PAP > 35 mmHg $(22,26,28)$. In diesen Fällen ist gemäß der Empfehlungen der ATS eine Lungentransplantation zu erwägen. Die Altersgrenze liegt dabei um 60 Jahre (2).

kurzgefasst: Die Pharmakotherapie wird ergänzt durch flankierende Maßnahmen, die nur individuell zum Einsatz kommen. Dazu zählen die Alpha-1-Antitrypsin-Substitutionstherapie, Impfungen, Sauerstofflangzeittherapie und operative Verfahren.

\section{Exazerbation der COPD}

Die COPD und hier insbesondere die schwereren Verlaufs- und Erkrankungsformen sind oftmals mit mehr oder weniger häufigen Exazerbationsphasen assoziiert. Meistens wird eine solche Exazerbation durch einen intrabronchialen/pulmonalen Infekt oder exogene Faktoren (z.B. Emissionen) ausgelöst und eine Verschlimmerung der Erkrankung i.S. eines Teufelskreises beschleunigt (Abb.2). Die Diagnosestellung einer solchen Exazerbation, die Ursachenfindung, die Abschätzung des Ausmaßes und die differentialdiagnostische Abklärung kann mit der in Teil 1 genannten Diagnostik erfolgen. Die Pharmakotherapie der Exazerbation basiert zunächst auf den schon genannten Substanzgruppen. Im Gegensatz zur chronischen COPD-Verlaufsform wird bei der Exazerbation die systemische Kortikosteroidtherapie (i.v. oder oral) empfohlen, da sie zu einer signifikant schnelleren Besserung der Lungenfunktion führt. Dies betrifft insbesondere Patienten mit einer $\mathrm{FEV}_{1}<50 \%$ des Sollwertes. Empfohlen sind Dosen von ca. $40 \mathrm{mg}$ oral Prednisolon/Tag (max. $100 \mathrm{mg}$ Prednisolon/Tag) über einen Therapiezeitraum von 1014 Tagen $(5,20)$. Eine längere Therapiedauer brachte in Bezug auf die Lungenfunktionsverbesserung und auch die Exazerbations-Rezidivrate keinen zusätzlichen klinischen Gewinn $(6,16)$.

\begin{tabular}{|c|c|c|c|c|}
\hline & Stufe 0 & Stufe I & Stufe II & Stufe III \\
\hline $\begin{array}{l}\text { Lungenfunktion } \\
\text { FEV }_{1 / \text { soll }} \\
\mathrm{FEV}_{1} / \mathrm{FVC}\end{array}$ & $\begin{array}{l}\text { Husten + } \\
\text { Auswurf }\end{array}$ & $\begin{array}{l}\geq 80 \% \\
<70 \%\end{array}$ & $\begin{array}{c}<80-\geq 30 \% \\
<70 \%\end{array}$ & $\begin{array}{l}<30 \% \\
<70 \%\end{array}$ \\
\hline Therapie & keine & \multicolumn{3}{|c|}{$\begin{array}{c}\text { kurzwirksame } \beta_{2} \text {-Antagonisten bei } \\
\text { Bedarf }\end{array}$} \\
\hline $\begin{array}{l}\text { zusätzlich }(\mathrm{II}+\mathrm{III}) \text { : } \\
\text { rehabilitative } \\
\text { Maßnahmen }\end{array}$ & & & \multicolumn{2}{|c|}{$\begin{array}{c}\text { Bronchodilatatoren } \\
\text { (Dauerth.: einzeln } \\
\text { und/oder Komb.) } \\
\text { inhalative Steroide } \\
\text { (Therapieeffekt } \\
\text { vorausgesetzt) }\end{array}$} \\
\hline $\begin{array}{l}\text { alle }(0-\text { III): } \\
\text { Risikovermeidung } \\
\text { Grippeimpfung }\end{array}$ & & & & $\begin{array}{l}\text { suppor- } \\
\text { tive Maß- } \\
\text { nahmen }\end{array}$ \\
\hline
\end{tabular}

Abb.1 Schweregradeinteilung und Therapiempfehlung zur Behandlung der COPD (modifiziert nach (20)).

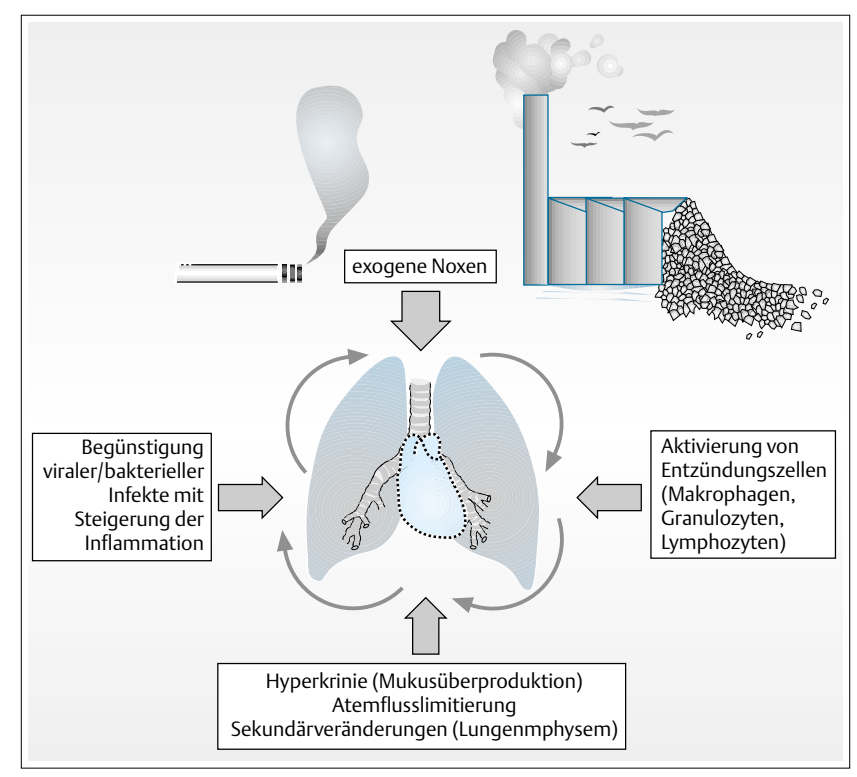

Abb.2 Der Teufelskreis von der COPD-auslösenden Exposition über die Hyperkrinie zur persistierenden Entzündungsreaktion im Bronchialepithel.

Bei einer Infektexazerbation wurden insbesondere folgende Keime isoliert: Streptococcus pneumoniae, Haemophilus influenza und Moraxella catarrhalis (10). Bei fortgeschrittenem Erkrankungsstadium und in Abhängigkeit zur Lungenfunktionsverschlechterung spielen gram-negative Erreger eine zunehmende Rolle (10). Ein purulentes Sputum korreliert zu einer signifikant erhöhten Keimzahl und erhöhten Entzündungsparametern. Eine erfolgreiche Antibiotikatherapie führt nicht nur zu einer Beseitigung des bakteriellen Erregers und zur klinischen Besserung, sondern auch zu einer Normalisierung der Inflammation (14). Eine kalkulierte Antibiotikatherapie, die nur bei Verdacht oder Nachweis einer bakteriellen Ursache indiziert ist, muss dieses Keimspektrum berücksichtigen $(11,24,25,27,30,33)$.

Die Sauerstofftherapie bildet den Eckstein supportiver Therapiemaßnahmen. Angestrebt wird ein $\mathrm{pO}_{2}$ von $>8,0 \mathrm{kPa}(=60$ $\mathrm{mmHg}$ ). $\mathrm{Zu}$ Beginn der Sauerstoffapplikation (meistens über Nasensonde) sollten die arteriellen und kapillär gemessenen Blutgase zu Beginn alle 30 Min. kontrolliert werden, um eine 
$\mathrm{CO}_{2}$-Retention oder Acidose frühzeitig erkennen und vermeiden zu können (9).

Die Pharmakotherapie während der COPD-Exazerbation wird insbesondere in der akuten Phase hochdosiert durchgeführt. Dazu können die verwendeten Kortikosteroide, $\beta_{2}$-Agonisten und das Theophyllin, in einer für Notfallsituationen entsprechend eingerichteten und erfahrenen Therapieeinheit (z.B. Krankenhaus) auch intravenös appliziert werden.

\section{Beatmungsverfahren}

Im Falle eines respiratorischen Versagens kann die nicht-invasive Beatmungsform eine invasive Beatmung vermeiden helfen. In Studien wurden Erfolgsraten bis zu 85\% beschrieben. Vorteile der nicht-invasiven Beatmung sind die Reduktion des $\mathrm{pCO}_{2}$ und die Steigerung des $\mathrm{pO}_{2}$ mit Reduktion des subjektiven Dyspnoeempfindens. Zudem verringert die nicht-invasive Beatmungsform die Krankenhausaufenthaltsdauer. Die Indikation für eine invasive mechanische Ventilationsform während einer COPDExazerbation basiert primär auf dem klinischen Eindruck (Schwere der Dyspnoe, Somnolenz, kardio-vaskuläre Komplikationen) und sekundär auf dem Ergebnis der Blutgasanalyse. Die invasive Beatmungsform erhöht das Mortalitäts- und Morbiditätsrisiko zum Beispiel an einer komplikativen Pneumonie ("ventilator associated pneumonia"). Zudem ist das "weaning" der Patienten schwierig $(1,5,20)$.

kurzgefasst: Die Therapie der Exazerbation besteht in einer intensivierten Gabe von bronchodilatativ wirkenden Medikementen, einer 10- bis 14-tägigen systemischen SteroidTherapie, im Falle eines bakteriellen Infektes aus einer kalkulierten Antibiotikagabe, die durch Sauerstoffgaben und bei respiratorischer Insuffizienz durch nicht-invasive und/ oder invasive Beatmungsverfahren ergänzt werden.

\section{Verlaufsbeobachtung}

Die COPD ist eine progressiv verlaufende Erkrankung, wobei sich die Lungenfunktion unabhängig von den ergriffenen therapeutischen Maßnahmen im Laufe der Jahre individuell schnell verschlechtern kann. In Abhängigkeit vom Schweregrad sollten die Patienten regelmäßig von einem Pneumologen gesehen werden. Wichtig sind die sorgfältige Anamnese, eine symptomorientierte Befunderhebung und die Durchführung einer Lungenfunktionsprüfung (mindestens eine Spirometrie). Bei einem $\mathrm{FEV}_{1}<40 \%$ des Sollwertes wird zusätzlich die Durchführung einer Blutgasanalyse in regelmäßigen $\mathrm{Ab}-$ ständen, z.B. ${ }^{1} / 4^{-}$oder $1 / 2$-jährlich empfohlen. Da die COPD-Patienten in der Regel schlecht und individuell unterschiedlich auf die Pharmakotherapie ansprechen, muss die Therapie im Hinblick auf ihre Effektivität und die zu erwartenden Nebenwirkungen regelmäßig überprüft und entsprechend angepasst werden (siehe insbesondere Therapie mit Glukokortikosteroiden). Ein wichtiges Maß für den Therapieerfolg ist die Reduktion der Exazerbationsrate, der typischen Symptome (Dyspnoe, Husten, Auswurf), die Steigerung der physischen Leistungsfähigkeit und die Vermeidung von Sekundärkomplikationen, wie dem Lungenemphysem, der respiratorischen Insuffizienz und dem Cor pulmonale $(1,5)$.

\section{Fazit für die Praxis}

Die Riskovermeidung (Abstinenz des Zigarettenrauchens) steht an erster Stelle der COPD-Therapie. Wesentliches therapeutisches Standbein der stabilen Erkrankungsform ist der Einsatz von Bronchodilatatoren, und nur bei entsprechendem Effektivitätsnachweis auch eine inhalative Steroidtherapie. Bei der akuten Exazerbation steht zusätzlich die zeitlimitierte systemische Steroidgabe, bei einer bakteriellen Infektion die kalkulierte Antibiotikatherapie, die das charakteristische Keimspektrum dieser Patienten berücksichtigen muss, und bei respiratorischer Insufizienz die Sauerstoffapplikation und/ oder Beatmungsverfahren im Vordergrund der therapeutischen Möglichkeiten.

\section{Literatur}

1 American Thoracic Society. Standards for the diagnosis and care of patients with chronic obstructive pulmonary disease. Am J Respir Crit Care Med 1995; 152: S77-S120

2 American Thoracic Society. International guidelines for the selection of lung transplant candidates. Am J Respir Crit Care Med 1998; 158: 335-339

3 Anthonisen NR, Connett JE, Kiley JP et al. Effects of smoking intervention and the use of an inhaled anticholinergic bronchodilator on the rate of decline of FEV 1 . The Lung Heath Study. JAMA 1994; 272: 1539-1541

4 Barnes PJ. Inhaled Corticosteroids are not beneficial in chronic obstructive pulmonary disease. Am J Respir Crit Care Med 2000; 161: 342-344

5 British Thoracic Society. Guidelines for the management of chronic obstructive pulmonary disease. Thorax 1997; 52: S1-S28

6 Bullard MJ, Liaw SJ, Tsai YH, Min HP. Early corticosteroid use in acute exacerbations of chronic airflow limitation. Am J Emerg Med 1996; 14: 139-143

7 Burge PS. EUROSCOP, ISOLDE and the Copenhagen City Lung Study. Thorax 1999; 54: 287-288

8 Burge PS, Calverley PMA, Jones PW, Anderson JA, Maslen TK. Randomised, double blind, placebo controlled study of fluticasone propionate in patients with moderate to severe chronic obstructiv pulmonary disease: the ISOLDE trial. BMJ 2000; 320: 1297-1303

9 Deutsche Gesellschaft für Pneumologie. Empfehlungen zur Sauerstoff-Langzeit-Therapie bei schwerer chronischer Hyoxämie. Pneumologie 1993; 47: 2-4

10 Eller J, Ede A, Schaberg T, Niederman MS, Mauch H, Lode H. Infective exazerbations of chronic obstructive pulmonary disease. Relation between bacteriologic etiology and lung function. Chest 1998; 113: 1542-1548

11 Ewig S, Merget R, Schäfer H, Bauer T, Lüderitz B. Therapie der akuten Exazerbation der chronisch-obstruktiven Lungenerkrankung. AtemwLungenkrkh 1998; 24: 18-27

12 Gillissen A. Kortisontherapie bei chronisch-obstruktiver Bronchitis Contra-Position. Dtsch Med Wochenschr 2000; 125: 222-223

13 Gillissen A. Steroideinsatz in der Langzeittherapie der COPD. MünchenDeisenhofen: Dustri-Verlag Dr. Karl Feistle, In: Scheuch G (Hrsg.). Aerosole in der Inhalationstherapie V. 2001: 34-43

14 Gompertz S, O'Brien C, Bayley DL, Hill SL, Stockley RA. Changes in bronchial inflammation during acute exacerbations of chronic bronchitis. Eur Respir J 2001; 17 : 1112-1119

15 van Grunsven PM, van Schayck CP, Derenne JP et al. Long term effects of inhaled corticosteroids in chronic obstructive pulmonary disease: a meta-analysis. Thorax 1999; 54: 7-14

16 Keatings VM, Jatakanon A, Worsdell YM, Barnes PJ. Effects of inhaled and oral glucocorticoids on inflammatory indices in asthma and COPD. Am J Respir Crit Care Med 1997; 155: 542-548

17 Leuenberger P, Anderhub HP, Brändli O et al. Management 1997 of chronic obstructive pulmonary disease. Schweiz Med Wochenschr 1997; 127: 766-782

18 National Emphysema Treatment Research Group. Patients at high risk of death after lung-volume-reduction surgery. N Engl J Med 2001; 345: 1075-1083

19 Paggiaro PL, Dahle R, Bakran I, Frith L, Hollingworth K, Efthimiou J. Multicentre randomised placebo-controlled trial of inhaled fluticasone propionate in patients with chronic obstructive pulmonary disease. Lancet 1998; 351: 773-780

20 Pauwels RA, Buist AS, Calverley PMA, Jenkins C, Hurd SS. Global Strategy for the Diagnosis, Management, and Prevention of Chronic Obstructive Pulmonary Disease. Am J Respir Crit Care Med 2001; 163: 1256-1276

21 Pauwels RA, Lofdahl C-G, Laitinen LA et al. Long-term treatment with inhaled budesonide in persons with mild chronic obstructive pulmonary disease who continue smoking. N Engl J Med 1999; 340: 1948-1953

22 Russi EW, Bloch KE, Weder W. Functional and morphological heterogeneity of emphysema and its implication for selection of patients for lung volume reduction surgery. Eur Respir J 1999; 14: 230-236

23 Russi EW, Stammberger U, Weder W. Lung volume reduction surgery for emphysema. Eur Respir J 1997; 10: 208-218 
24 Schaberg T, Dalhoff K, Ewig S, Lorenz J, Wilkens H. Deutsche Gesellschaft für Pneumologie: Empfehlungen zur Therapie der ambulant erworbenen Pneumonie. Pneumologie 1998; 52: 450-462

25 Schäfer H, Ewig S, Gillissen A. Therapie der schweren Exazerbation bei chronisch obstruktiver Lungenerkrankung (COPD). Intensivmed 2000; im Druck

26 Schulz C, Riedel M, Gillissen A, Emslander HP. Präoperative Identifizierung des pulmonalen Risikopatienten vor Lungenresektion. AtemwLungenkrkh 1998; 24: 205-214

27 Soler N, Torres A, Ewig S et al. Bronchial microbial patterns in severe exacerbations of chronic obstructive pulmonary disease (COPD) requiring mechanical ventilation. Am J Respir Crit Care Med 1998; 157: 1498-1505

28 Thurnheer R, Bingisser R, Stammberg $U$ et al. Effect of lung volume reduction surgery on pulmonary hemodynamics in severe pulmonary emphysema. Eur J Cardiothorac Surg 1998; 13: 253-258

29 Vestbo I, Sørensen T, Langer P, Brix A, Torre P, Viskum K. Long-term effect of inhaled budesonide in mild and moderate chronic obstructive pulmonary disease: a randomised controlled trial. Lancet 1999; 355: 1819-1823

30 Vogel F, Worth H, Adam D et al. Rationale Therapie bakterieller Atemwegsinfektionen. Empfehlung der Paul-Ehrlich-Gesellschaft für Chemotherapie und der Deutschen Atemweosliga Chemotherapie J 2000: 9: 3-23

31 Vogelmeier C. Kortisontherapie bei chronisch-obstruktiver Bronchitis Pro-Position. Dtsch Med Wochenschr 2000; 125: 220-221
32 Wettengel R, Böhning W, Cegla U et al. Empfehlungen der Deutschen Atemwegsliga zur Behandlung von Patienten mit chronisch obstruktiver Bronchitis und Lungenemphysem. Med Klinik 1995; 90: 3-7

33 Worth H, Adam D, Handrick W et al. Prophylaxe und Therapie von bronchiale Infektionen. Med Klinik 1997; 12: 699-704

\section{Korrespondenz}

Prof. Dr. med. A. Gillissen

Robert Koch-Klinik

Städtisches Klinikum "St. Georg"

Nikolai-Rumjanzew-Straße 100

04207 Leipzig

Tel.: 0341/4231202

Fax: 0341/4231203

E-Mail: adrian.gillissen@sanktgeorg.de

Eingang des Manuskriptes: 10.12.2001

Annahme der revidierten Fassung zur Publikation: 28.1.2002

\section{Der konkrete Fall}

Der örtlich und zeitlich orientierte 63-jährige Patient wurde uns von einem niedergelassenen Pneumologen zugewiesen. Er klagte über eine seit ca. 5-6 Jahren langsam zunehmende belastungsabhängige Dyspnoe. Seit seiner Jugendzeit hat er täglich etwa eine Packung Zigaretten geraucht, sodass sich eine kumulative Zigarettenmenge von $>40$ pack years errechnete. Seitens seines Berufes sei er nie einer wesentlichen Staubexposition ausgesetzt gewesen. Seit den letzten Wochen hatte die Dyspnoe jedoch deutlich an Schwere zugenommen, sodass die kleinste Anstrengung, z.B. Zähneputzen oder der Gang zur Toilette, zur Belastung geworden seien. Zudem würde Husten und meist klarer, gelegentlich aber auch eitriger Auswurf bestehen.

Klinischer Befund: Der übergewichtige Patient (Größe $174 \mathrm{~cm}$, Gewicht $93 \mathrm{~kg}$ ) wies eine Lippenzyanose auf, die Auskultation des Thorax erbrachte ein leises Atemgeräusch und leise Herztöne. Der Klopfschall war über beiden Lungen hypersonor, und es bestanden diskrete Knöchel- und prätibiale Ödeme.

Diagmostilk: In der a.p. Röntgenaufnahme ließ sich eine dekompensierte Herzinsuffizienz als Ursache für die geklagte Dyspnoe ausschließen. Die Rarefizierung der Lungengefäße und die tiefstehenden Zwerchfelle wiesen auf eine deutliche Überblähung hin, wie sie für ein Lungenemphysem typisch ist. Die Lungenfunktion zeigte eine schwergradige, unter inhalativer Gabe eines kurzwirksamen $\beta_{2}$-Rezeptoragonisten und eines Parasympatholytikums (Ipratropiumbromid) nicht wesentlich beeinflussbare $\left(\mathrm{FEV}_{1}:<15 \%,<150 \mathrm{ml}\right)$ Atemwegsobstruktion. Der in der Flussvolumenkurve sichtbare Knick im exspiratorischen Schenkel wies auf einen Bronchiolenkol- laps hin, wie er für ein Emphysem typisch ist. In der Bodyplethysmographie stellte sich in der Druckvolumenkurve die typische Keulenform, wie bei einer Atemwegsobstruktion dar. Die kapillär gemessene Blutgasanalyse belegte eine respiratorische Globalinsuffizienz $\left(\mathrm{PO}_{2} 45 \mathrm{mmHg}, \mathrm{PCO}_{2} 56\right.$ $\mathrm{mmHg}$ ), an die sich der wache Patient offenbar adaptiert hatte. Ergänzend wurde eine Computertomographie der Lunge angefertigt, die keinen Zweifel an dem ausgeprägten klein- bis mittelgradig ausgeprägten, ubiquitär in beiden Lungenhälften vorhandenen Emphysem ließ.

Therapie: Die initiale Therapie bestand in der zunächst hochdosiert intravenös applizierten Theophyllin- und Glukokortikosteroid-Gabe, die nach 14 Tagen abgeschlossen wurde. Zusätzlich erhielt der Patient inhalativ kurzwirksame $\beta_{2}$-Agonisten bei Bedarf, einen langwirksamen $\beta_{2}$-Agonisten, Ipratropiumbromid und Steroide als Dauermedikation. Die Effektivität der inhalativen Steroidmedikation wird nach Entlassung aus der stationären Betreuung nach ca. ${ }^{1} / 4$ Jahr kontrolliert und die Steroidindikation entsprechend angepasst. Physiotherapeutische Maßnahmen zur Optimierung der Atemtechnik, Schulungen zum Erlernen der Ursachen und des Umgangs mit der Erkrankung, Anleitung zur korrekten Anwendung der inhalativen Medikation und Raucherentwöhnungsmaßnahmen ergänzten die Pharmakotherapie.

Diskussion: Der Patient litt an einem ausgeprägten Lungenemphysem und einer schwergradigen nicht-reversiblen Atemwegsobstruktion. In den allermeisten Fällen lässt sich die COPD klinisch, durch die Lungenfunktion und die Blutgasanalyse diagnostizieren, sodass die bildgebenden Verfahren einschließlich des CT-Thorax oder der Echokardiographie nur in Zweifelsfäl- len zur Abklärung der Differentialdiagnosen oder zur Quantifizierung von Sekundärkomplikationen (z.B. Rechtsherzinsuffizienz, wie bei unserem Patienten klinisch angedeutet) indiziert sind. Die Ursache dieses Befundes liegt ausschließlich in dem ausgeprägten jahrzehntelangen Nikotinabusus. Der Patient bot einen typischen klinischen Verlauf, da a) die COPD nicht zuletzt auch aufgrund des ausgeprägten CT-morphologischen Befundes sicher länger als die geklagten 5-6 Jahre bestanden haben dürfte, b) die Patienten oft über Jahre bis Jahrzehnte ihre Beschwerden negieren, und c) die Erkrankung in der Regel über lange Zeiträume schleichend verläuft und sich die Patienten an diesen Verlauf adaptieren.

Die Pharmakotherapie hat bei solchen Fällen nur eine begrenzte Wirkung, da sich die Lungenfunktion im Gegensatz zum Asthma bronchiale durch die lange Erkrankungsdauer und Expositionszeit nur schwer beeinflussen und die Exazerbationsraten nur unzureichend reduzieren lassen. Die dem Patienten zusätzlich offerierte Sauerstofflangzeittherapie wird, eine $\mathrm{O}_{2}$-Gabe von > $16 \mathrm{~h} /$ Tag vorausgesetzt, sein Mortaliätsrisiko senken. Als weitere konservative Therapiemaßnahme kämen noch nicht-invasive Beatmungsformen in Frage, deren Langzeiteffekt auf Morbidität und Mortalität allerdings derzeit noch nicht abschließend beurteilbar sind. Ergänzend dazu werden rehabilitative Maßnahmen, Impfungen (Influenza, Pneumokokken), Optimierung des Ernährungsstatus, da Untergewicht mit einer erhöhten Mortalität bei COPD assoziiert ist (betrifft nicht den hier vorgestellten Fall), und insbesondere die vollständige Einschränkung des Zigarettenabusus empfohlen.

Prof. Dr. med. Adrian Gillissen Leipzig 